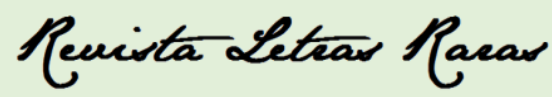

ISSN: 2317-2347 - Vol. 5, Ano 5, № 3 - 2016

\title{
La pédagogie de projet en cours de FLE : expérience dans un collège brésilien
}

\section{Project-based learning and FFL: a brazilian junior high school case study}

Jean-François Brunelière*

Clarissa Laus Pereira Oliveira ${ }^{* *}$

\begin{abstract}
RÉSUMÉ
La pédagogie de projet est souvent présentée comme un aboutissement de ce que pourrait actuellement être la didactique de l'enseignement. Dans la perspective diachronique de l'enseignement du Français Langue Etrangère (FLE), c'est une méthodologie qui se propose d'aller au-delà des principes proposés dans la perspective actionnelle, en partant du contexte et les motivations réelles de l'apprenant. Cet article propose quelques réflexions à partir du cas du projet « Nous parlons français », qui permet à des étudiants brésiliens de collège et de lycée de suivre des cours de FLE de manière volontaire dans certains établissements de la ville de Florianópolis, au sud du Brésil. Nous présentons la manière dont une classe de sixième a pu définir de manière démocratique et collaborative son projet d'étude pour un semestre, comment celui-ci a été organisé et le résultat final qui a été produit. La participation active et la motivation sont les deux facteurs principaux qui sous-tendent toute la dynamique de groupe et le résultat final du projet en termes d'apprentissages comportementaux (savoir-être) et linguistiques (savoirs et savoir-faire).
\end{abstract}

MOTS-CLES : FLE ; Pédagogie de projet ; Collège; Brésil.

\begin{abstract}
Project-based learning is often presented as what should be the didactics of teaching nowadays. In a diachronic perspective of the teaching of French as a Foreign Language (FFL), it is a methodology which proposes to go beyond the principles proposed in the languagelaction perspective, with a perspective based on the context and the real motivations of the student. This article proposes some reflections based on the project "Nous parlons français", which allows Brazilian junior high school and high school students to take FFL courses on a voluntary basis in Florianópolis, Brazil. We discuss how a sixth class was able to define in a democratic and collaborative way its own semester project, how it was organized and the final result produced. Active participation and motivation are the two main factors underlying both group dynamics and the final result in terms of behavioral (interpersonal skills) and linguistic (knowledge and know-how) learning.
\end{abstract}

KEYWORDS : FFL; Project-based learning; Junior high school ; Brazil

\section{Introduction}

Pour s'adapter aux évolutions permanentes de la société le système scolaire est amené à se rénover périodiquement. Entre la révolution industrielle du $\mathrm{XIX}^{\mathrm{e}}$ siècle, le mouvement de mondialisation qui a suivi la Seconde Guerre mondiale et l'apparition de nouvelles technologies

\footnotetext{
* Etudiant du cours de licence en Lettres (français) de l'Université Fédérale de Santa Catarina (UFSC) - Email : jean.gradfrances@gmail.com.

${ }^{* *}$ Docteure en littérature comparée de l'Université Fédérale de Rio Grande do Sul (UFRGS) et professeure de Méthodologie de l'enseignement du français au Département de Méthodologie de l'enseignement (MEN) de l’Université Fédérale de Santa Catarina (UFSC) - Email : clarissa.oliveira@ufsc.br.
} 


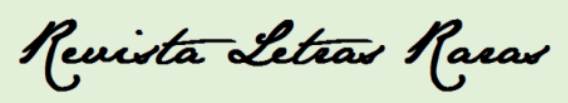

ISSN: 2317-2347 - Vol. 5, Ano 5, № 3 - 2016

à l'origine d'une explosion de la communication internationalisée sur Internet et les réseaux sociaux, il ne fait aucun doute que l'école a dû évoluer. Dans ce contexte, les méthodologies d'enseignement des langues étrangères font également l'objet de réformes, de nouvelles propositions venant compléter ou substituer celles qui leur préexistaient. En ce qui concerne le Français Langue Etrangère (FLE dorénavant), et malgré l'éclectisme (PUREN, 1994) qui caractérise la période récente, c'est la pédagogie de projet (encore appelée apprentissage par projet ou méthodologie de projet) qui représente désormais l'approche la plus novatrice. Nous présenterons donc cette pédagogie dans une perspective diachronique permettant d'en distinguer les principales spécificités par rapport aux méthodologies précédentes, avant de décrire sa mise en œuvre dans le cadre de cours de FLE dans un collège de Florianópolis (Brésil) en 2016. Nous poursuivrons cette discussion par quelques réflexions relatives aux avantages et limitations de la pédagogie de projet dans le cadre particulier des cours de FLE au collège.

\section{Méthodologies du FLE : de la méthode traditionnelle à la pédagogie de projet}

L'enseignement et l'apprentissage des langues étrangères n'a évidemment pas attendu l'accélération de la mondialisation qui a suivi la Seconde Guerre mondiale pour élaborer des méthodologies. Les spécialistes de l'étude historique des méthodologies ont été amenés à effectuer des regroupements de ces dernières, selon la fréquence et l'ampleur de leur utilisation, et à définir des « périodes méthodologiques » qui, même si leurs limites temporelles et la vision de pratique hégémonique qu'elles transmettent sont toujours discutables, permettent de dégager une vision d'ensemble de la perspective diachronique de l'utilisation des méthodes d'enseignement des langues étrangères en général, et du FLE en particulier. Il y a un certain consensus entre les différents auteurs sur la progression générale qui a été suivie par les méthodologies depuis le XIX ${ }^{\mathrm{e}}$ siècle. Ainsi, selon Puren (1988 ; 1994), Martinez (1999) et Leffa (1988 ; 2012), la succession des grandes périodes méthodologiques peut se résumer ainsi : la méthodologie traditionnelle (encore appelée «traduction et grammaire »), calquée sur l'apprentissage, très analytique, des langues mortes, basée sur l'utilisation de modèles et attribuant un rôle important au professeur, a été largement utilisée jusqu'au début du $\mathrm{XX}^{\mathrm{e}}$ siècle, époque à laquelle la méthode directe va lui succéder - dans les programmes d'enseignement scolaire officiels, mais en réalité les deux méthodes, traditionnelles et directes, existent depuis 


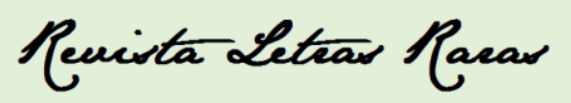

ISSN: 2317-2347 - Vol. 5, Ano 5, № 3 - 2016

l'antiquité de manière parallèle. Cette méthode prévoit une utilisation exclusive de la langue étrangère - donc sans recours à la traduction - et privilégie l'oral et la qualité de la prononciation, tout en augmentant la participation active de l'apprenant. La méthode audioorale qui suit la Seconde Guerre mondiale, s'appuie sur les études naissantes dans la linguistique appliquée et la psychologie pour, en partant de situations bien identifiées, développer la compréhension et la production orales par l'utilisation d'exercices de répétitions. Les avancées technologiques favoriseront par la suite un passage à la méthode audio-visuelle, qui multipliera les possibilités de présentation de situations les plus diverses, en insistant sur l'authenticité et l'utilisation de la langue étrangère uniquement, tout en continuant à privilégier l'oralité. L'apparition de l'approche communicative est une conséquence de la politique d'intégration européenne et de l'augmentation de la circulation des personnes. On perçoit alors la nécessité d'atteindre des niveaux minimaux de communications entre les individus amenés à se rencontrer et que la langue a avant tout une fonction : plus qu'un système à connaître et maîtriser, elle permet surtout d'échanger avec des interlocuteurs et est essentiellement performative. Il est donc possible de travailler, via des simulations, les différentes situations qu'un apprenant pourra rencontrer et acquérir certains automatismes dans l'emploi de formules ou de vocabulaire spécifiques. Le matériel utilisé doit être authentique et les genres les plus divers sont recherchés. Mais la situation, bien qu'impliquant de fait l'apprenant dans une situation de communication, n'a pas nécessairement de lien direct avec la réalité quotidienne de celui-ci et l'on peut paradoxalement travailler sur des situations de «communication » complètement virtuelles pour l'apprenant, qui ne les a jamais rencontrées - et peut-être ne les rencontrera jamais. C'est en grande partie pour répondre à cette difficulté que la perspective actionnelle est apparue au début des années 2000, avec la mise en place du Cadre Européen Commun de Référence pour les Langues (CECRL dorénavant), qui la présente ainsi :

La perspective privilégiée ici est, très généralement aussi, de type actionnel en ce qu'elle considère avant tout l'usager et l'apprenant d'une langue comme des acteurs sociaux ayant à accomplir des tâches (qui ne sont pas seulement langagières) dans des circonstances et un environnement donné, à l'intérieur d'un domaine d'action particulier. Si les actes de parole se réalisent dans des activités langagières, celles-ci s'inscrivent elles-mêmes à l'intérieur d'actions en contexte social qui seules leur donnent leur pleine signification. (CONSEIL DE L'EUROPE, 2001, p. 15) 


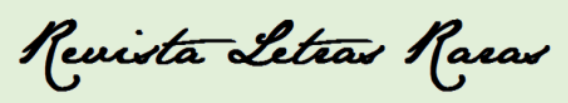

ISSN: 2317-2347 - Vol. 5, Ano 5, № 3 - 2016

On observe que cette perspective prend en compte, au-delà des tâches à réaliser, l'ensemble des aspects contextuels qui permettent à l'apprenant de situer son usage de la langue et de se comporter d'une manière moins artificielle.

La dernière évolution méthodologique que nous souhaitons évoquer est la pédagogie de projet. S'agissant de l'objet de notre étude de cas, nous lui consacrons une présentation plus détaillée. On peut faire remonter ses origines au travaux du philosophe américain John Dewey qui, au début du $\mathrm{XX}^{\mathrm{e}}$ siècle, perçoit la nécessité d'éviter de dissocier théorie et pratique et propose donc d'inscrire la construction de la connaissance dans l'expérience. Il suggère alors la mise en place d'une école « expérimentale », qui se rapproche d'un véritable laboratoire. Selon Dewey (1915, p. 296, notre traduction), «les avantages moraux d'une forme active d'éducation renforcent ses bénéfices intellectuels ${ }^{1}$, puisqu'ils préparent l'individu à une véritable transformation de la société. Ainsi, à partir des intérêts et motivations de l'apprenant, l'éducation se produit au moyen d'expériences, avec des changements fréquents de responsables, choisis parmi les élèves, au long des diverses activités réalisées pour mener à bien le projet. Plus qu'une simple manière de fournir des contenus, l'éducation vise à former des citoyens préparés à agir démocratiquement afin de transformer la société à laquelle ils participent. Bien que l'on trouve déjà beaucoup des principes de la pédagogie de projet actuelle dans cette conception de l'apprentissage présentée par Dewey, il faudra près d'un siècle pour qu'ils soient repensés dans l'optique de l'enseignement des langues étrangères. Blumfield et al. (1991, p. 371) définissent de manière synthétique ce qu'est l'essence d'un projet lorsqu'ils affirment que :

[i]l y a deux composants fondamentaux pour les projets : ils demandent une question, ou un problème, qui sert à organiser et diriger les activités ; et ces activités aboutissent à une série de produits, avec un produit final qui répond à la question initiale ${ }^{2}$. (BLUMFIELD et al. (1991, p. 371- Notre traduction).

Proulx (2004) précise un peu les choses quelques années plus tard, en définissant l'apprentissage par projet comme :

\footnotetext{
${ }^{1}$ Dans l'original : The moral advantages of an active form of education reenforce its intellectual benefits.

${ }^{2}$ Dans l'original : [t]here are two essential components of projects: They require a question or problem that serves to organize and drive activities; and these activities result in a series of artifacts, or products, that culminate in a final product that addresses the driving question.
} 


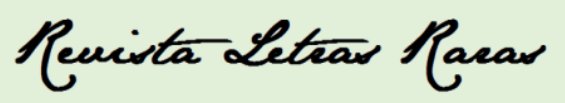

ISSN: 2317-2347 - Vol. 5, Ano 5, № 3 - 2016

[...] un processus systématique d'acquisition et de transfert de connaissances au cours duquel l'apprenant anticipe, planifie et réalise, dans un temps déterminé, seul ou avec des pairs et sous la supervision d'un enseignant, une activité observable qui résulte, dans un contexte pédagogique, en un produit fini évaluable. (PROULX, 2004, p. 31)

Notons que si l'initiative de l'élaboration de la question initiale, ainsi que la production finale et son évaluation, peuvent être laissées aux apprenants, cela ne signifie pas que le rôle du professeur soit réduit à celui d'un simple spectateur. Si l'apprenant est le "chef de file" de sa formation, l'enseignant est le "vigile" (PROULX, 2004, p. 10).

La pédagogie de projet implique un travail qui est par nature flexible. Elle ne prévoit donc pas a priori quels outils seront utilisés. On pourra donc envisager de mettre en œuvre des activités qui sont généralement associées à d'autres méthodologies, allant de l'échange de correspondances avec des classes d'autres pays, à la rédaction de journaux en passant par des observations en plein air - ces types d'activités étant habituellement associées aux techniques dites « Freinet » (1964). Le niveau de complexité du projet dépasse néanmoins celui de simples activités, aussi intéressantes et bien construites soient-elles. Le projet doit en effet permettre aux apprenants de s'organiser pour travailler dans des configurations de groupe variables (de l'individuel au grand groupe en passant par des petits groupes) et de mettre en œuvre des compétences diversifiées, allant au-delà de celles qui sont liées aux fonctions cognitives immédiates, dans une perspective d'ensemble structurée. Par ailleurs, dans le cadre d'un projet en FLE on veillera, en parallèle de la mise en œuvre de diverses compétences, à ce que les activités qui permettent de mener à bien le projet fassent intervenir le maximum de compétences linguistiques. Sur ce point, des études montrent déjà que l'absence de matériel didactique préélaboré (un manuel présentant une séquence organisée - mais fixe - de contenus) ne représente pas un obstacle à une progression correcte de l'expression des apprenants en langue étrangère (SCHLEMMINGER, 2003). C'est au professeur d'organiser l' "apparition » des outils linguistiques au moment où ils sont nécessaires dans le projet, en évitant les contretemps qui risqueraient de perturber les apprenants. Les situations que le projet peut faire surgir étant quasi illimitées, il convient que le professeur anticipe au maximum les différentes éventualités pour que des difficultés imprévues ne viennent pas stopper l'élan des apprenants en situation de découverte/résolution autonome. On perçoit bien que le professeur, privé des supports didactiques traditionnels qu'il pourrait étudier à l'avance, doit envisager d'autres manières de préparer son cours, et se montrer flexible face aux directions parfois imprévisibles que peut 


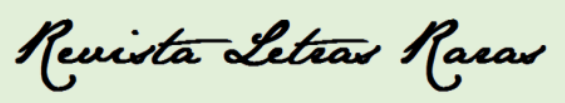

ISSN: 2317-2347 - Vol. 5, Ano 5, № 3 - 2016

prendre le projet, confié en grande partie aux apprenants. Loin d'être un spectateur passif du déroulement du projet, dont il se déresponsabiliserait, il doit au contraire réfléchir en permanence aux apports qu'il sera amené à faire et à la manière d'accompagner les apprenants vers la réussite du projet, qui se matérialisera - au moins en partie, puisque d'autres aspects sont également en jeu - dans la production finale.

Une fois les principes de la pédagogie de projet énoncés, reste à vérifier comment se passent les choses lorsque l'on met en place cette méthodologie de manière concrète dans une classe, et dans notre cas : dans une classe de FLE.

\section{Exemple de mise en œuvre de la pédagogie de projet dans une classe de FLE d'un collège brésilien}

Nous avons choisi de mettre en œuvre la pédagogie de projet dans le cadre d'un stage, qui fait partie de la formation obligatoire du professeur dans le cadre de la licence en Lettres (option français) de l'Université Fédérale de Santa Catarina (UFSC). Les élèves en cours de licence sont amenés à intervenir pour donner des cours dans différents collèges et lycées de la ville de Florianópolis. Sous la supervision de leur professeure, ils préparent le plan d'enseignement pour un semestre et le plan de chaque cours, puis sont évalués sur la manière dont ils gèrent leurs cours et sur leurs rapports d'activités. Certains collèges et lycées adhèrent, de manière volontaire, à un projet qui leur offre la possibilité d'inclure des cours de français dans leur établissement. Ce projet, appelé « Nous parlons français », est inscrit comme projet d'extension de l'UFSC, ce qui permet aux élèves participants de recevoir des certificats pour leur participation en fin de semestre. Il est également soutenu par l'Association des Professeurs de Français de Santa Catarina (APFSC). Les élèves des collèges et lycées qui participent au cours du projet « Nous parlons français » le font sur leur temps libre, par exemple le matin, s'ils ont leurs cours habituels des autres matières du collège ou du lycée l'après-midi. Le projet « Nous parlons français » n'utilise pas de manuel didactique ; les cours sont construits «surmesure » pour chaque classe. L'expérience dont nous rendons compte dans cet article s'est produite avec un groupe d'une dizaine d'élèves de $6^{\text {ème }}\left(6^{\circ}\right.$ ano $)$ dans un collège de Florianópolis, au cours du premier semestre de l'année 2016. Ces élèves, âgés de 11 et 12 ans, n'avaient pas de connaissance préalable du français et un rapide questionnaire initial a permis de vérifier qu'ils n'avaient aucun contact avec cette langue dans leur vie quotidienne (pas 


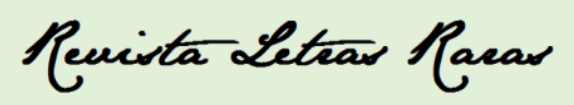

ISSN: 2317-2347 - Vol. 5, Ano 5, № 3 - 2016

d'intérêt particulier pour la musique ou le cinéma francophone, pas de parents francophones, aucune consultation de sites francophones sur Internet).

La manière de mettre en œuvre la pédagogie de projet dans cette classe peut être synthétisée en quelques étapes clés. La première étape est en réalité une étape préalable au démarrage des activités. Il s'agit de se réunir avec les élèves et de leur expliquer la manière de travailler qui sera adoptée pour le cours de français. Nous considérons que cette étape est indispensable au bon fonctionnement du projet, car elle permet à des élèves, généralement peu habitués à décider des activités qu'ils réalisent en classe, de comprendre qu'ils peuvent réellement influencer la manière dont vont se produire les cours de français. Proulx souligne l'importance de cette étape, lorsqu'il déclare (2004, p. 93) que les apprenants « doivent savoir et comprendre quelles intentions pédagogiques animent leur enseignant pour qu'il leur propose cette approche. ». Il ajoute encore « Non seulement doivent-ils les comprendre, mais aussi y souscrire. » (PROULX, 2004, p. 93), pour indiquer que les élèves aussi doivent croire en cette pédagogie. C'est pourquoi, en fin de présentation, nous avons aussi demandé aux élèves s'ils imaginaient qu'ils pourraient apprendre le français en travaillant de cette manière, pour tester, en toute honnêteté, leur adhésion au projet. Ils ont alors manifesté leur enthousiasme par rapport à la méthodologie proposée. Ils n'ont pu le faire de manière valide qu'après avoir reçu les informations nécessaires. Lors de la phase d'explication, les grands moments du projet sont donc présentés, sans bien entendu pouvoir, à cet instant, dire précisément en quoi consistera le projet, qui n'a pas encore été défini. Mais on présente son organisation générale en distinguant le moment de sa définition (étape d'élaboration d'une problématique), la phase de recherche sur le sujet, puis en indiquant que tout ce travail fera l'objet d'un rendu final, dont la forme reste à déterminer. Cette discussion initiale permet d'éveiller la curiosité des élèves, qui commencent à percevoir qu'ils pourront proposer des choses, mais aussi de les responsabiliser puisqu'ils comprennent qu'ils devront également travailler à la fois individuellement et en groupe et que ce travail ne servira pas uniquement à la classe, mais sera également «montré » (diffusé) à l'extérieur de celle-ci.

La seconde étape importante, qui a lieu dès le premier cours, après la phase d'explication initiale du fonctionnement général du cours, consiste à définir la problématique qui sera travaillée tout au long du semestre. A ce moment précis, le professeur ne sait pas encore quelle sera la problématique travaillée, le choix appartenant réellement aux élèves. Dans le cas du groupe avec lequel nous avons travaillé durant ce stage, la méthode retenue pour faire 


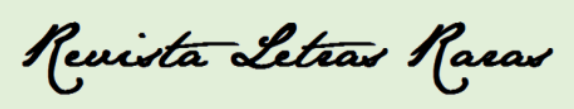

ISSN: 2317-2347 - Vol. 5, Ano 5, № 3 - 2016

émerger une problématique allant au-delà d'une simple thématique a été de proposer aux élèves de se réunir par groupe de trois et de noter (en portugais, puisque les élèves n'ont pas les éléments linguistiques pour expliquer cela en français) les questions qui les intéressent le plus, dans leur vie « réelle », sans que cela n'ait nécessairement de lien avec l'école. La présentation de la problématique sous forme de question, plutôt que le simple énoncé d'un thème, oriente déjà la suite du travail vers un mode de production axé sur la recherche. Si au lieu de penser à étudier « les abeilles », par exemple, les élèves proposent de répondre à la question « pourquoi les abeilles ont-elles besoin de produire du miel ? », la réflexion est déjà en marche et les perspectives d'explorations à partir de cette question sont démultipliées. Afin de permettre aux élèves de passer naturellement du niveau de la thématique au niveau de la problématique nous avons réalisé une petite activité sur les différents types de question qui existent, ce qui a également permis de commencer à travailler les mots interrogatifs en français. Après cet «échauffement » à créer des questions, les élèves ont commencé à produire leurs propres questions en petit groupes. Bien que cela n'ait pas été sollicité, plusieurs questions soulevées par les élèves avaient un lien direct avec la culture française, les Français ou la France, probablement en raison de la nouveauté que représentait pour ces élèves la présence d'un cours de français dans leur collège. D’autres questions étaient néanmoins liées à des sujets très différents, qui traduisent soit un intérêt personnel de l'élève («Comment sont apparus les chiffres?»), soit la présence d'une thématique dans l'actualité locale («Quels sont les nouveaux sports qui seront présents aux jeux olympiques ? », à quelques semaines des jeux de Rio de Janeiro). Une fois les premiers centres d'intérêt identifiés, nous avons choisi de définir une question commune pour le grand groupe, afin que le maximum d'élèves s'identifie à la problématique du projet. Pour cela nous avons procédé par vote. C'est finalement une question liée à la langue française qui a été retenue par le groupe : «Quelle est l'origine du français ?». Bien que cette question soit extrêmement intéressante, elle n'est pas évidente à aborder avec un groupe de cet âge. Afin de mieux préparer la suite du projet et d'envisager différents axes d'approches nous avons immédiatement repris cette question en l'écrivant au centre du tableau et travaillé avec le grand groupe pour créer une carte mentale à partie de cette question. Les élèves étaient libres de s'exprimer en portugais, mais tous les éléments étaient reformulés et discutés en français avant d'être notés au tableau pour former la carte mentale. Cette transition entre les deux langues permet à la fois aux élèves de ne pas être limités dans leur créativité à ce moment du cours (premier contact avec la langue et moment de définition du projet du semestre) 


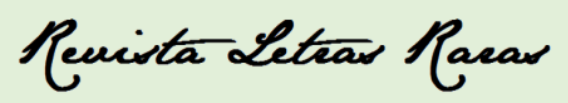

ISSN: 2317-2347 - Vol. 5, Ano 5, № 3 - 2016

et de commencer à se familiariser avec la langue française. Une fois les grands axes de recherche identifiés, reste à définir comment organiser le projet. Selon le niveau d'étude et l'âge des élèves, leur implication dans la conception de la méthodologie de recherche est plus ou moins sollicitée. Dans le cas d'élèves de $6^{\text {ème }}$, débutants complets en français, il serait contreproductif de leur confier la responsabilité totale d'une recherche autonome sur un sujet aussi complexe que celui qui a été choisi par le groupe. Aussi avons-nous pensé à un développement du projet en trois temps.

Le projet développé en 2016 par ce groupe d'élèves de $6^{\text {ème }}$ s'articule autour de trois axes : le premier vise à construire les éléments de base permettant de répondre à la question du projet, le second vise à construire l'apprentissage linguistique en français, le dernier vise enfin à produire un résultat qui puisse être présenté au-delà de la classe. Pour répondre au premier point, sans que les élèvent ne risquent de se décourager face à une recherche complexe qu'ils ne pourraient mener en français, le rôle du professeur a consisté à proposer aux élèves une démarche qui leur permette à la fois d'effectuer une recherche correspondant à leur niveau scolaire (non seulement en français, mais également dans les autres domaines qui interviennent dans la problématique retenue : Histoire et Géographie en particulier) et de mettre en œuvre des dynamiques de groupes. Ces dernières ont été conçues de manière à faire intervenir différentes tailles de groupes et avec l'objectif de ne pas répéter les activités, de manière à ce que la progression du grand groupe dépende des résultats de chaque petit groupe. Pour cela nous avons élaboré des séries d'activités, confiées aux petits groupes (binômes à ce moment). Ces activités consistaient à construire, petit à petit, un panorama géographique et historique (en remontant au temps des Gaulois), permettant aux élèves de comprendre comment un peuple est associé à une ou plusieurs langues, comment ces langues circulent, naissent ou disparaissent. De nombreux supports visuels ont dû être utilisés car les élèves de $6^{\text {ème }}$ n'avaient pas encore étudié, en cours d'Histoire ou de Géographie, toutes les périodes et concepts que nous devions utiliser. Par ailleurs, afin de créer une dynamique et une émulation entre les élèves, nous avons élaboré une version du « jeu de l'oie » dans laquelle les groupes devaient avancer le plus rapidement possible en répondant à tour de rôle aux questions. Cette ressource - «artificielle » puisque toute la présentation des activités aurait pu avoir lieu sans le jeu - a donné un surplus de motivation aux différents groupes. Profitons de cette remarque pour indiquer que rien n'interdit d'utiliser des activités ludiques au cours d'un projet. La pédagogie de projet n'impose pas en effet de faire uniquement de la recherche pure. On peut même envisager une utilisation de jeux 


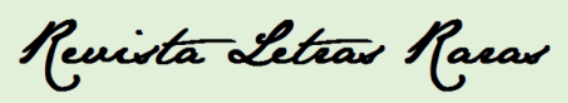

ISSN: 2317-2347 - Vol. 5, Ano 5, № 3 - 2016

vidéo - tels que les « jeux sérieux » (SANCHEZ ; NEY ; LABAT, 2011) par exemple - sans que la dynamique générale en soit affectée, pour peu que les activités ludiques intègrent des éléments pertinents par rapport à la logique du projet considéré. Le second axe, spécifique aux éléments linguistiques, nécessite de prévoir un apport solide et organisé de contenus, sans toutefois que ceux-ci ne deviennent l'objectif principal de l'activité. Si des contenus lexicaux et grammaticaux élémentaires - tels que les noms de pays, de langues, quelques verbes, les chiffres et les articles par exemple - sont relativement simples à faire intervenir dans la dynamique générale, les éléments plus structuraux, tels que la conjugaison ou encore la syntaxe de la phrase doivent être travaillés de manière plus spécifique, au moyen de quelques exercices oraux d'accompagnement et des moments de «consolidation linguistique » où le professeur vérifie avec les élèves leur compréhension de quelques notions. Le troisième axe concerne enfin la production finale. Une fois que les élèves s'étaient donné les moyens de répondre à leur problématique initiale sur l'histoire de la langue française - et avaient par ailleurs également compris le lien entre cette langue et le portugais, parlé au Portugal et au Brésil, par un mécanisme de comparaison/différenciation assimilé très naturellement par les élèves, sans que l'aide du professeur ne soit nécessaire - est venu le moment de penser à la forme que pourrait prendre la présentation finale. Maintenant que les élèves avaient une meilleure perception de la langue et, de nouveau, à partir des observations qu'ils avaient pu faire au fil des cours - puisque le projet s'écrit avec la classe - les élèves ont pu penser à une présentation relative au français aujourd'hui et dans le contexte de Florianópolis. Au cours du semestre, nous avions par exemple eu l'occasion d'étudier les couleurs et les élèves s'étaient aperçus que celles-ci apparaissaient parfois, en français, dans le nom de certains commerces brésiliens ; il a donc été assez naturel de penser à montrer cela aux autres élèves du collège. Mais, encore une fois, avant de se lancer dans cette activité, il convenait d'en faire un véritable projet. Le groupe a ainsi défini une stratégie, de regroupement des mots en français par catégories et de recherche de vocabulaire, puisque le lexique des élèves était encore très limité. Une réflexion sur le sujet des mots d'emprunts a permis d'identifier une liste de mots pouvant servir de base à une première discussion: la liste des mots étrangers ayant une orthographe «adaptée » en portugais, disponible sur le site du Sénat brésilien (SÉNAT, 2015). A partir de ces premiers exemples le grand groupe a établi des catégories de mots étrangers que l'on pouvait rencontrer dans la langue portugaise et dans l'espace public de la ville (par exemple dans les vitrines des magasins, les noms donnés aux immeubles, etc.). C'est à partir de ces catégories que le grand groupe s'est 


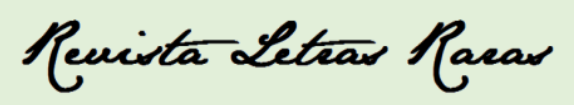

ISSN: 2317-2347 - Vol. 5, Ano 5, № 3 - 2016

organisé pour répartir les tâches, chaque élève étant responsable d'identifier, dans son quartier ou aux alentours du collège, les mots en français qu'il pouvait rencontrer et les classer dans les différentes catégories choisies par la classe. Après des séances de vérification (et en particulier l'exclusion des mots identifiés à tort comme français par les élèves, en raison de leur consonnance), le groupe a enfin pu passer à la conception d'une affiche. Il a ensuite été proposé de mettre cette affiche en évidence dans l'école, avec l'autorisation et le soutien de la direction. Pour autant, le projet ne se termine pas avec la présentation du résultat. Les élèves sont encore amenés à réfléchir à leur progression au long du semestre. Un questionnaire individuel est donc proposé aux élèves lors de la dernière rencontre du semestre. Il prévoit des questions spécifiques pour les différents types d'activités réalisées, sur l'autoévaluation de la participation, des comportements et des acquis. Plus que les résultats absolus qu'il peut fournir, ce questionnaire permet surtout aux élèves de prendre du recul par rapport à ce qu'ils ont pu réaliser tout au long du semestre et de prendre conscience de leur progression.

\section{Limitations et avantages de la méthode projet en cours de FLE}

L'expérience acquise au cours de ce semestre de mise en œuvre de la pédagogie de projet nous permet de poursuivre notre réflexion sur quelques difficultés rencontrées dans le cadre de son application au FLE et sur les divers avantages qu'elle présente.

Une première difficulté, assez facilement corrigeable, est due au propre enthousiasme des élèves et au fait de travailler en langue étrangère. Pris dans la réalisation du projet, ils peuvent se concentrer les résultats partiels du projet en oubliant un peu de travailler en français chaque fois que c'est possible. Il convient donc parfois que le professeur se rappelle au souvenir $\mathrm{du}$ groupe et organise la recherche des apports linguistiques, ainsi que des phases de « consolidation», réellement dédiées aux questions de langue. Ceci peut s'organiser assez naturellement sous la forme de rappels et d'activités dédiées périodiques. La principale difficulté que nous avons pu ressentir lors de la mise en œuvre de la pédagogie de projet tient cependant au niveau linguistique du groupe. Malgré un accueil très positif, le choix de la problématique était un véritable défi pour un groupe de débutants en français. Elle serait probablement traitée plus facilement par un groupe plus avancé. L'autonomie dans la recherche était limitée avec le groupe considéré et la sélection des matériels a dû être très minutieuse 


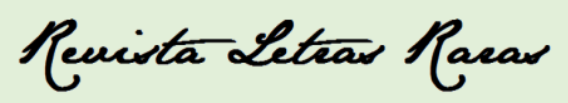

ISSN: 2317-2347 - Vol. 5, Ano 5, № 3 - 2016

(choix de matériel didactique authentique mais pour des groupes d'âge inférieur, avec beaucoup d'illustrations et des termes «transparents »). Certains matériels ont même dû être totalement créés, par exemple pour regrouper des périodes historiques généralement présentées indépendamment. Certains éléments ont par ailleurs été «détournés » pour travailler des questions linguistiques (partir des Gaulois pour décrire leurs caractéristiques physiques et les couleurs de ce qu'ils portent par exemple). Il va de soi qu'un projet réalisé dans la langue maternelle des élèves, sur un même sujet, aurait permis de traiter des aspects complémentaires. Une autre difficulté tient au niveau des connaissances des élèves de $6^{\text {ème }}$ dans d'autres disciplines (particulièrement en Histoire et en Géographie dans notre cas, mais également dans leur propre langue maternelle, en ce qui concerne par exemple le métalangage grammatical (savoir ce qu'est un verbe ou un article)). Les élèves ont certainement beaucoup appris, mais la progression a dû prendre en compte les apports nécessaires à chaque étape. Il a ainsi fallu comprendre qui étaient les Gaulois, par une évolution progressive des élèves qui sont passés, de manière autonome, au vu des éléments qu'ils découvraient (époque, vêtements, outils, habitudes), de leur réalité à celle de l'époque, sur un autre continent, en allant du concept d' « indiens », à celui de «vikings portugais » pour se rapprocher d'une compréhension de l'identité gauloise.

Ce dernier exemple permet d'évoquer immédiatement l'un des avantages de la pédagogie de projet : les élèves apprennent de manière plus naturelle, sans se limiter aux frontières d'une unique discipline. Leur intelligence se déploie partout où le projet les emmène, jusqu'où ils ont besoin d'aller pour répondre à leurs questions. Ils ne perçoivent d'ailleurs pas réellement les difficultés des concepts qu'ils abordent, ni même de la langue, tant ils sont emportés par la dynamique et leur curiosité. Les élèves sont réellement portés par les dynamiques des différentes configurations de groupes, soucieux de contribuer au résultat global. Ces observations correspondent bien à ce que constate Schlemminger (2003, p. 49) lorsqu'il indique que la pédagogie de projet favorise les apprentissages et acquisitions de compétences complexes, de même qu'elle implique la mise en œuvre d'organisations collectives complexes. Le professeur, un peu plus présent au début du projet, le temps que les élèves s'habituent à leur propre autonomie et prennent confiance, peut s'appuyer de plus en plus sur le groupe. On voit également surgir des comportements qui sont en général peu favorisés par un travail organisé plus «mécaniquement » par le professeur, tel celui de cette élève qui a commencé spontanément à distribuer les tâches et à demander à ses camarades de 


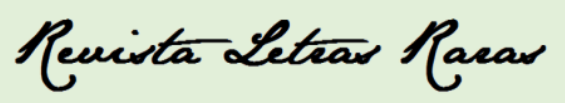

ISSN: 2317-2347 - Vol. 5, Ano 5, № 3 - 2016

lui envoyer les photos qu'ils prenaient par email pour qu'elle les regroupe et les transmette au professeur. Ces exemples de prise d'initiative sont surprenants au début, mais on peut gager qu'ils se reproduiront de plus en plus souvent au fur et à mesure que les élèves prendront l'habitude d'être véritablement acteurs de leur apprentissage. Si le professeur n'est certainement pas dispensé de vérifier que le calendrier général - et en particulier celui de la production finale - est respecté, il sait qu'il peut compter sur le groupe pour proposer des ajustements en cours de projet. La flexibilité est d'ailleurs l'un des principaux intérêts de cette pédagogie. Sans programme formel précis à respecter, au-delà des compétences linguistiques dont l'étude et la pratique a été prévue en début de semestre, la créativité des élèves peut s'exprimer et les activités être élaborées en fonction de l'avancement réel du projet. Les élèves prennent ainsi conscience petit à petit que la proposition initiale n'était pas feinte et qu'ils sont réellement responsables de définir bon nombre d'éléments de leur programme d'étude. La motivation initiale de commencer à étudier une nouvelle langue, renforcée par la rencontre des défis proposés par les différentes phases du projet, est consolidée par la confiance qu'ils acquièrent lorsqu'ils voient qu'ils sont réellement en mesure de définir la manière de présenter leur produit final. Si la motivation et l'augmentation de la responsabilité sont mis en avant par divers auteurs qui s'intéressent à la pédagogie de projet (cf. PROULX, 2004, p. 58-62 par exemple), son aptitude à développer des compétences de conduite de projets collectifs est également souvent relevée (PERRENOUD, 1999 ; PROULX, 2004). Ainsi, au-delà de «simples» savoir, la pédagogie de projet permet aux élèves de développer un savoir-faire lié aux compétences de recherche et d'orgaisation, ainsi qu'un véritable savoir être, lié au travail en groupe. Notons enfin que la motivation ne se limite pas aux élèves ; le fait de pouvoir présenter un produit à l'ensemble de l'établissement scolaire incite également les responsables de sa direction à valoriser le travail réalisé dans le cadre du cours de FLE. Ce dernier élément est essentiel pour l'avenir du cours, lorsqu'on se souvient qu'il s'agit d'une activité basée sur le volontariat et que le français n'est pas obligatoire dans les écoles de beaucoup d'Etats brésiliens.

\section{Considérations finales sur les conditions de réussite de la pédagogie de projet}

Nous souhaitons discuter, pour conclure, quelques éléments ponctuels qui nous semblent favoriser le déroulement d'un projet pour des groupes d'élèves de la tranche d'âge 


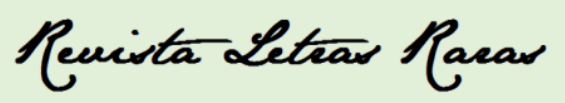

ISSN: 2317-2347 - Vol. 5, Ano 5, № 3 - 2016

considérée (11-12 ans). Tout d'abord, et puisque la pédagogie de projet le permet, sous réserve que l'esprit général du projet soit respecté, nous recommandons l'insertion de quelques activités ludiques, en « couverture » des apprentissages et mise en pratiques de compétences et éléments linguistiques. En effet, pour peu qu'elles soient « réaliste»- et ne se résument pas à un semblant de jeu inintéressant, qui ne motivera pas l'élève -, ce type d'activité permet à l'élève d'oublier la notion d' « exercice » et apporte un surplus de motivation à la participation. Les élèves de cet âge cherchent alors visiblement à participer et à se dépasser, plus que dans un simple tour de table sans enjeu, qui conviendrait peut-être à des adultes, plus prompts à réaliser l'activité «pour elle-même ». Un autre aspect important est la relation avec le reste des membres de l'établissement scolaire. Il est évident que de tels projets sont l'occasion idéale pour établir des ponts entre disciplines et travailler de manière conjointe avec d'autres professeurs. La flexibilité de la pédagogie de projet permet de nombreuses adaptations, y compris à des exigences qui lui sont a priori étrangères, comme par exemple un système d'évaluations systématiques par examens écrits, ainsi que cela est pratiqué dans le collège où nous avons mis en place cette pédagogie en cours de FLE. Nous avons ainsi pu profiter des phases de «consolidation linguistiques » intégrées à notre projet pour réaliser une « révision » qui a précédé un « examen écrit » traditionnel, sans que cela ne remette en cause le déroulement du projet, ni ne perturbe la compréhension de la dynamique générale, de la part des élèves. Ces phases d'évaluation formelle nous ont par ailleurs permis de vérifier et de démontrer aux conseillers pédagogiques que les élèves assimilaient beaucoup de notions linguistiques au cours du projet. Ceci est essentiel car, comme le souligne Reverdy (2013), l'appui de l'établissement qui l'accueille conditionne le succès de la pédagogie de projet.

\section{Références}

BLUMFIELD et al. Motivating project-based learning: Sustaining the doing, supporting the learning. Educational Psychologist, v. 26, n.3-4, p. 369-398, 1991.

CONSEIL DE L'EUROPE. Cadre européen commun de référence pour d'apprentissage et l'enseignement des langues. Strasbourg. Paris: Didier, 2001.

DEWEY, John. Schools of Tomorrow. New York: Dutton, 1915.

FREINET, Célestin. Les techniques Freinet de l'École moderne. Paris: Armand Colin, 1964. 


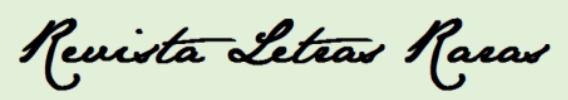

ISSN: 2317-2347 - Vol. 5, Ano 5, № 3 - 2016

LEFFA, Vilson. Metodologia do ensino de línguas In: BOHN, Hilário; VANDRESEN, Paulino (org.). Tópicos em linguística aplicada: o ensino de línguas estrangeiras. Florianópolis: UFSC, 1988. p. 211-236

. Ensino de línguas: passado, presente e futuro. Revista de Estudos da Linguagem, v. 20, n.2, p. 389-411, 2012

MARTINEZ, Pierre. Didática de línguas estrangeiras. São Paulo: Parábola Editorial, 2009

PERRENOUD, Philippe. Apprendre à l'école à travers des projets : pourquoi ? comment ?, 1999. Disponible sur :

<http://www.unige.ch/fapse/SSE/teachers/perrenoud/php_main/php_1999/1999_17.html>.

Consulté le 13 oct. 2016.

PROULX, Jean. Apprentissage par projet. Sainte-Foy : Presses de l'Université du Québec, 2004.

PUREN, Christian. Histoire des méthodologies de l'enseignement des langues. Paris : Clé International, 1988.

. La didactique des langues étrangères à la croisée des méthodes. Essai sur l'éclectisme. Paris : CRÉDIF-Didier, 1994.

REVERDY, Catherine. Des projets pour mieux apprendre ? Institut Français d'Education. Dossier d'actualité. Veille et Analyses, n 82, p. 1-24, 2013.

SANCHEZ, Eric; NEY, Muriel; LABAT, Jean-Marc. Jeux sérieux et pédagogie universitaire: de la conception à l'évaluation des apprentissages. Revue internationale des technologies en pédagogie universitaire, v.8, n.1-2, 2011, p. 48-57.

SCHLEMMINGER, Gerald. La pédagogie Freinet et le travail en projet. Les langues modernes, n.3, 2003. p. 47-57

SÉNAT (site du sénat brésilien). Lista de palavras que já estão aportuguesadas. 2015.

Disponible sur : <www12.senado.leg.br/manualdecomunicacao/redacao-e-estilo/estilo/listade-palavras-que-ja-foram-aportuguesadas>. Consulté le 12 avril 2016.

Recebido em : 19/11/2016

Aceito em : 30/11/2016 